\title{
Sex- and Age-Related Differences in the Distribution of Metastases in Patients With Upper Urinary Tract Urothelial Carcinoma
}

\author{
Marina Deuker, MD ${ }^{1,2}$; Giuseppe Rosiello, MD ${ }^{1,3}$; Lara Franziska Stolzenbach, MD ${ }^{1,4}$; Thomas Martin, $\mathrm{MD}^{1}$; \\ Claudia Collà Ruvolo, MD ${ }^{1,5}$; Luigi Nocera, MD1,3; Zhe Tian; Frederik C. Roos, MD²; Andreas Becker, MD²; \\ Luis A. Kluth, MD²; Derya Tilki, MD'; Shahrokh F. Shariat, MD ${ }^{6,7,8}$; Fred Saad, MD; \\ Felix K.H. Chun, MD²; and Pierre I. Karakiewicz, $\mathrm{MD}^{1}$
}

\section{ABSTRACT}

Background: The distribution of metastatic sites in upper tract urothelial carcinoma (UTUC) is not well-known. Consequently, the effects of sex and age on the location of metastases is also unknown. This study sought to investigate age- and sex-related differences in the distribution of metastases in patients with UTUC. Materials and Methods: Within the Nationwide Inpatient Sample database (2000-2015), we identified 1,340 patients with metastatic UTUC. Sites of metastasis were assessed according to age $(\leq 63,64-72$, $73-79$, and $\geq 80$ years) and sex. Comparison was performed with trend and chi-square tests. Results: Of 1,340 patients with metastatic UTUC, 790 (59.0\%) were men (median age, 71 years) and 550 $(41.0 \%)$ were women (median age, 74 years). The lung was the most common site of metastases in men and women $(28.2 \%$ and $26.4 \%$, respectively), followed by bone in men ( $22.3 \%$ vs $18.0 \%$ of women) and liver in women ( $24.4 \%$ vs $20.5 \%$ of men). Increasing age was associated with decreasing rates of brain metastasis in men (from $6.5 \%$ to $2.9 \% ; P=.03$ ) and women (from $5.9 \%$ to $0.7 \%$; $P=.01$ ). Moreover, increasing age in women, but not in men, was associated with decreasing rates of lung (from $33.3 \%$ to $24.3 \%$; $P=.02$ ), lymph node (from $28.9 \%$ to $15.8 \% ; P=.01$ ), and bone metastases (from $22.2 \%$ to $10.5 \% ; P=.02$ ). Finally, rates of metastases in multiple organs did not vary with age or sex (65.2\% in men vs $66.5 \%$ in women). Conclusions: Lung, bone, and liver metastases are the most common metastatic sites in both sexes. However, the distribution of metastases varies according to sex and age. These observations apply to everyday clinical practice and may be used, for example, to advocate for universal bone imaging in patients with UTUC. Moreover, our findings may also be used for design considerations of randomized trials.

J Natl Compr Canc Netw 2021;19(5):534-540 doi: $10.6004 /$ jnccn.2020.7637

${ }^{1}$ Cancer Prognostics and Health Outcomes Unit, Division of Urology, University of Montreal Health Center, Montreal, Quebec, Canada; ${ }^{2}$ Department of Urology, University Hospital Frankfurt, Frankfurt am Main, Germany;

${ }^{3}$ Department of Urology and Division of Experimental Oncology, Urological Research Institute, IBCAS San Raffaele Scientific Institute, Milan, Italy; ${ }^{4}$ MartiniKlinik Prostate Cancer Center, University Hospital Hamburg-Eppendorf, Hamburg, Germany; ${ }^{5}$ Department of Urology, University of Naples Federico II, Naples, Italy; ${ }^{6}$ Department of Urology, Comprehensive Cancer Center, Medical University of Vienna, Vienna, Austria; ${ }^{7}$ Institute of Urology and Reproductive Health, I.M. Sechenov First Moscow State Medical University, Moscow, Russia; and ${ }^{8}$ Department of Urology, University of Jordan, Amman, Jordan.

\section{Background}

Upper tract urothelial carcinoma (UTUC) represents a rare entity, as evidenced by its contribution to only $5 \%$ to $10 \%$ of urothelial cancers. ${ }^{1}$ Due to its rarity, no large-scale observational data are available to describe the distribution of metastatic sites in these patients, especially if age and sex stratifications are considered. Only 2 previous studies, which were based on 52 and 337 patients, respectively, addressed the distribution of metastatic sites. ${ }^{2,3}$ Shinagare et $\mathrm{al}^{2}$ found no difference in the metastatic pattern for different tumor stages, histologic groups, or locations. Dong et $\mathrm{al}^{3}$ assessed cancer-specific and overall survival according to metastatic site and found that surgical resection of the primary tumor might still lead to survival benefits for highly selected patients with metastases.

The importance of stratification according to age and sex has been suggested in other studies, ${ }^{4,5}$ but it has never been applied to a sufficiently large sample of patients with UTUC. However, in everyday clinical practice and in the planning and design of randomized controlled trials, knowledge of distribution and potential age- and sex-specific differences in metastatic UTUC sites is important. We addressed this point and tested for distribution and variability in the distribution of metastases according to age and sex in a contemporary cohort of patients with metastatic UTUC. Specifically, we hypothesized that the distribution of metastases varies according to sex, and further postulated that age affects distribution patterns differently in men and women.

\section{Materials and Methods}

\section{Study Population}

This study relied on the Nationwide Inpatient Sample (NIS) database (2000-2015), which is composed of longitudinal

See JNCCN.org for supplemental online content. 
hospital inpatient databases from the Healthcare Cost and Utilization Project and includes $20 \%$ of US inpatient hospitalizations. ${ }^{6}$ Within the NIS, we focused on patients aged $>18$ years with the primary diagnosis of metastatic UTUC (ICD-9-CM codes 189.1 and 189.2) in 2000 through 2015 (supplemental eFigure 1, available with this article at JNCCN.org). Locations of metastatic sites were identified using ICD-9-CM codes as follows: lung (197.0), mediastinum (197.1), pleura (197.2), other respiratory organs (trachea, bronchus; 197.3), small intestine including duodenum (197.4), large intestine and rectum (197.5), peritoneum and retroperitoneum (197.6), liver (197.7), other digestive organs (esophagus, gallbladder, pancreas, stomach), spleen (197.8), regional lymph nodes (196.2, 196.6), distant lymph nodes (196.0, 196.1, 196.3, 196.5, 196.8, 196.9), kidney (198.0), urinary tract (198.1), skin (198.2), brain (198.3), other parts of the nervous system (198.4), bone (198.5), ovary (198.6), adrenal gland (198.7), breast (198.81), and genital organs (198.82). Patients with exclusive metastases in the urinary tract and kidney were excluded based on the notion that invasion per continuitatem and synchronous or metachronous urinary lesions are common in UTUC and do not account for distant metastasis criteria. Overall, these criteria yielded 1,340 (790 men and 550 women) evaluable patients (unweighted population).

\section{Variable Definition}

Age was categorized according to quartiles: $\leq 63,64-72$, $73-79$, and $\geq 80$ years. According to previously established methodology, ${ }^{5,7}$ abdominal metastases included liver, adrenal gland, peritoneum/retroperitoneum, kidney, colorectal, duodenum, ovary, spleen, other digestive system, and urinary tract. Similarly, thoracic metastases included lung, pleura, mediastinum, bronchus, and trachea. Conversely, brain and bone metastases were considered as 2 independent sites. Other variables included number of metastatic sites at the time of diagnosis (1 vs $\geq 2$ sites).

\section{Statistical Analyses}

Descriptive statistics included frequencies and proportions for categorical variables. Means, medians, and ranges were reported for continuously coded variables. Chi-square, Kruskal-Wallis, and $t$ tests examined the statistical significance of differences in proportions, medians, and means, respectively. Five sets of analyses were performed. First, we investigated rates of site-specific metastases within each sex group. Second, we investigated rates of site-specific metastases according to age group and sex. Third, the effect of age and year of diagnosis on the distribution of metastatic sites was assessed by trend tests according to sex. Fourth, we investigated the rates of single versus multiple metastatic sites according to sex and age categories. Fifth, we investigated the median age at diagnosis per sex according to different metastatic sites. Finally, we investigated the rates of abdominal, thoracic, and brain metastases in a conditional fashion, according to sex. A stepwise model assessed rates of thoracic metastases in either the absence or presence of abdominal metastases and rates of brain metastases in either the absence or presence of thoracic metastases.

$\mathrm{R}$ version 3.4.3 (R Foundation for Statistical Computing) was used for all statistical analyses. All tests were 2 -sided with the significance level set at $P<.05$.

\section{Results}

General Characteristics of the Study Population Overall, 1,340 patients with metastatic UTUC in 2000 through 2015 were identified. Of those, 790 (59.0\%) were men and $550(41.0 \%)$ were women. The primary site of UTUC in the renal pelvis versus the ureter was not statistically different between men (496 [62.8\%] vs 294 [37.2\%], respectively) and women (322 [58.5\%] vs 228 [41.5\%], respectively). A statistically significant difference $(P=.04)$ existed with respect to age at diagnosis. Women were diagnosed at a median age of 74 years (interquartile range [IQR], 64-81 years) versus 71 years among men (IQR, 63-78 years).

\section{Location of Metastases According to Sex}

Figure 1 depicts rates of site-specific metastases according to sex. Lung metastases were most common in both sexes (28.2\% vs $26.4 \%$ ). In men, the second most common metastatic site was bone $(22.3 \%$ of men vs $18.0 \%$ of women [fourth most common site]) versus the liver in women $(24.4 \%$ of women vs $20.5 \%$ of men [fourth most common site]). Lymph node metastasis ranked third in both sexes (21.9\% in men vs $22.5 \%$ in women). Combined retroperitoneal and peritoneal metastases $(15.7 \%$ in men vs $16.5 \%$ in women) versus adrenal metastases $(6.6 \%$ vs $5.6 \%$, respectively) ranked fifth and sixth, respectively. Finally, brain metastases were rare and were identified in $4.2 \%$ of men and $2.4 \%$ of women (eighth in men and tenth in women). None of these sex-related differences achieved statistical significance.

\section{Location of Metastases According to Age and Sex}

Increasing age was associated with decreasing rates of brain metastasis in men (from $6.5 \%$ to $2.9 \% ; P=.03$ ) and women (from $5.9 \%$ to $0.7 \% ; P=.01$ ) (Table 1 ). In women, increasing age was associated with decreasing rates of lung (from $33.3 \%$ to $24.3 \%$; $P=.02$ ), lymph node (from $28.9 \%$ to $15.8 \%$; $P=.01$ ), and bone (from $22.2 \%$ to $10.5 \%$; $P=.02)$ metastases. All other metastases did not show significant changes in rates according to age. In an additional analysis evaluating rates of metastatic sites 


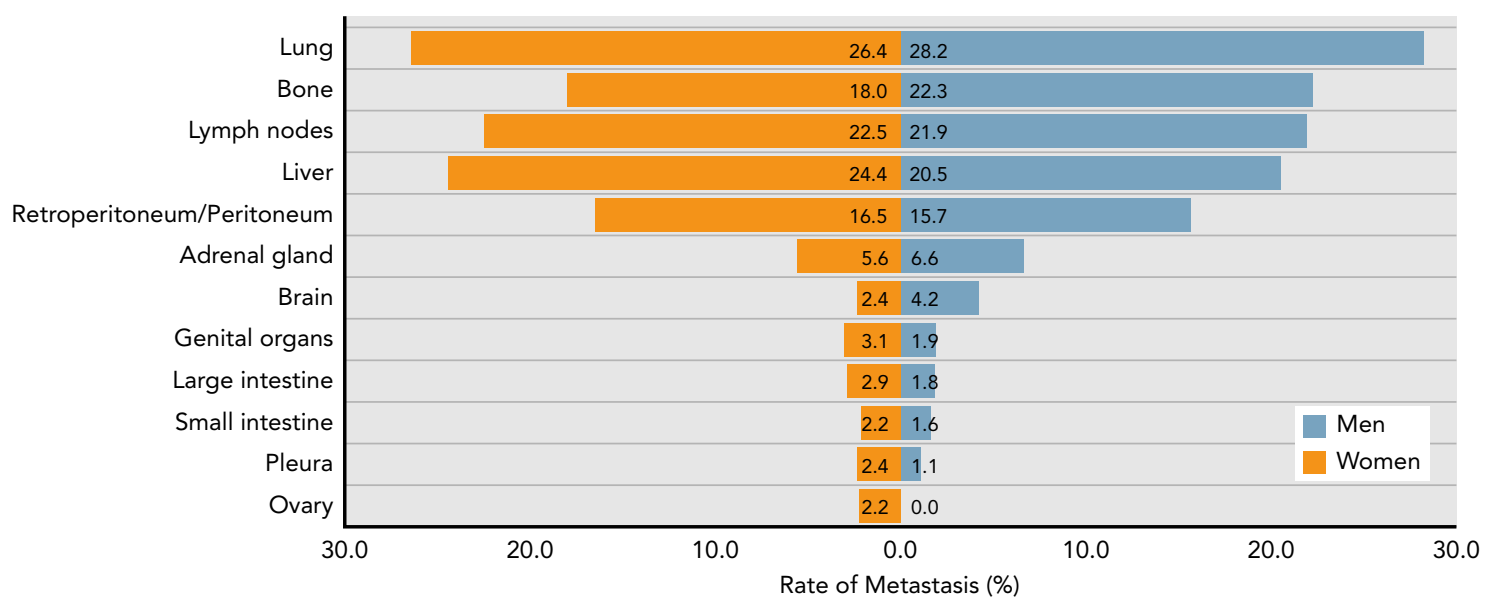

Figure 1. Rates of metastatic sites in 1,340 patients (790 men and 550 women; unweighted population) with metastatic upper tract urothelial carcinoma.

stratified according to year of diagnosis, we did not find any meaningful changes over time.

\section{Effects of Sex and Age on Specific Solitary \\ Metastatic Sites}

Overall, 515 men (65.2\%) and 366 women (66.5\%) had $\geq 2$ metastatic sites. In age-stratified, site-specific analyses, we focused on lung (exclusive lung vs lung combined with other metastases), bone (exclusive vs combined), liver (exclusive vs combined), and brain (exclusive vs combined). Only exclusive liver metastases showed a clinically meaningful and statistically significant increase of exclusive liver metastases rates in women with increasing age (from $21 \%$ to $41 \%$; $P=.04$ ). Among all other comparisons, no significant changes were recorded (data not shown).

\section{Age According to Different Metastatic Sites and Sex}

Figure 2 presents the relationship between age at diagnosis according to different metastatic sites and sex. Patients with brain metastases were substantially younger (median age, 62 years in women and 67 years men), whereas those with metastases at all other sites presented with a median age of $\geq 70$ years. The median age of female patients with abdominal metastases (74.5 years) was significantly higher than that of female patients with brain metastases (62 years; $P=.04$ ). All other comparisons did not yield significant differences in age according to sex and metastatic site.

\section{Conditional Patterns of Metastatic Distribution of \\ Brain Metastases}

Finally, we examined the conditional prevalence of brain metastases after stratification according to the presence or absence of abdominal and thoracic metastases. In the absence of thoracic and concomitant abdominal metastases, brain metastases were recorded in $3.6 \%$ of patients. Conversely, in the opposite scenario, with presence of abdominal and concomitant thoracic metastases, brain metastases were recorded in $<7 \%(\mathrm{n}<11)$. Similarly, in the presence of either abdominal or thoracic metastases, brain metastases were present in $<2 \%$ to $7.9 \%$ of patients, respectively (Figure 3 ).

\section{Discussion}

No single large-scale study ${ }^{2}$ has reported on the distribution of UTUC metastases. Consequently, the effects of sex and age on the location of metastases is also unknown. Based on this unmet need, we investigated sites of metastases according to sex and age within a large, contemporary, population-based dataset. Specifically, we hypothesized that a sex-related effect of age may exist in the distribution of metastases in patients with UTUC. Our study produced several important observations.

First, our study provides the first large-scale tabulation of metastases according to their location in UTUC. Specifically, abdominal and thoracic imaging is invariably recommended and performed in individuals considered at risk for metastatic UTUC. ${ }^{8}$ This approach is consistent with the distribution of all metastatic sites except for bone metastases. Notably, bone metastases ranked second in men and fourth in women. Consequently, standard imaging should include bone, which may not be imaged routinely in all of its extent with abdominal CT imaging. In light of our findings, routine bone surveys may be considered in all patients. Alternatively, bone scans may be used. However, predominance of lytic lesions may limit their usefulness because not all lytic lesions are reliably identified in bone scans. ${ }^{9,10}$ This observation is not reflected in the most contemporary imaging guidelines for UTUC. Currently, the NCCN Clinical Practice Guidelines in Oncology (NCCN Guidelines) for Bladder Cancer ${ }^{11}$ recommend bone imaging in patients with UTUC in the presence of bone 


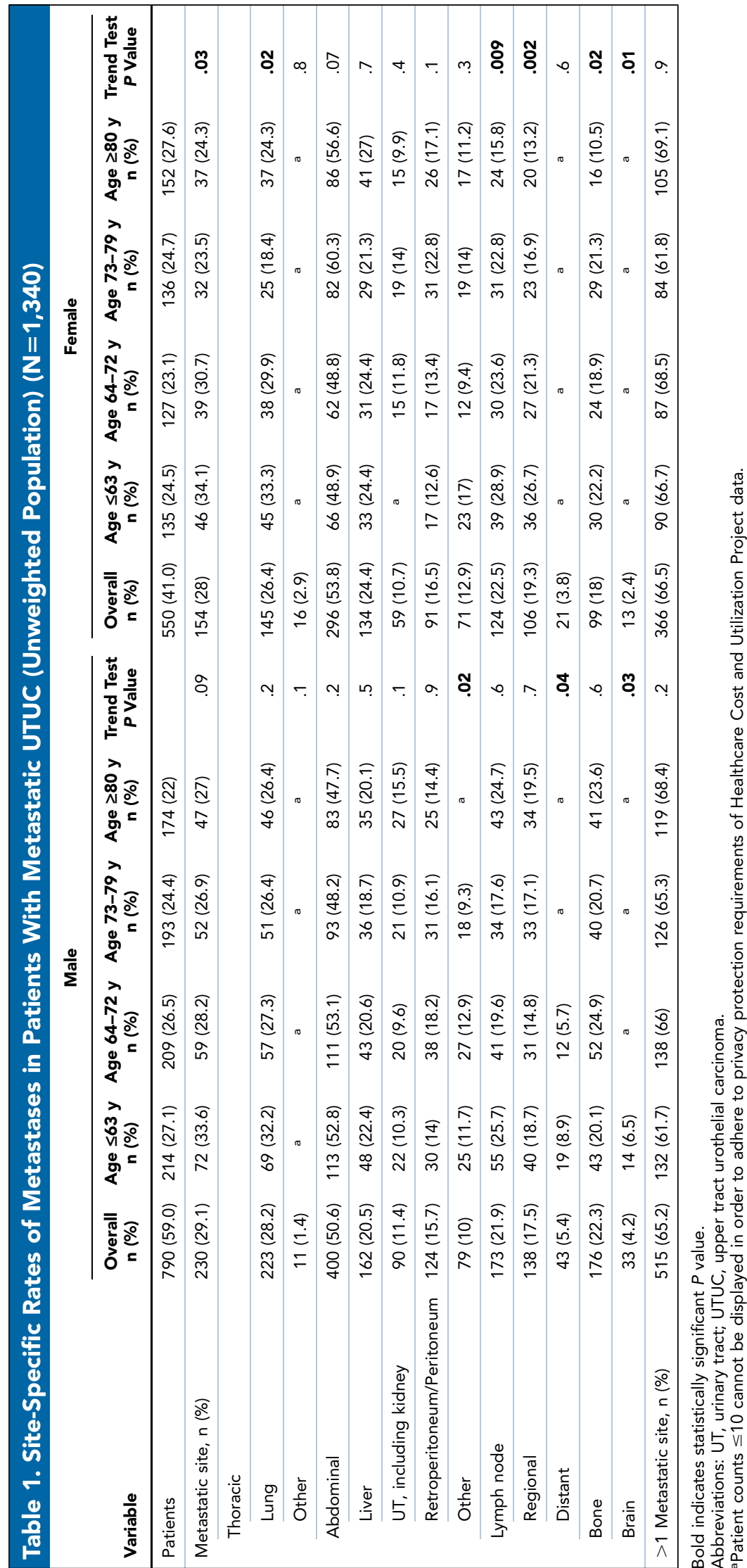


A

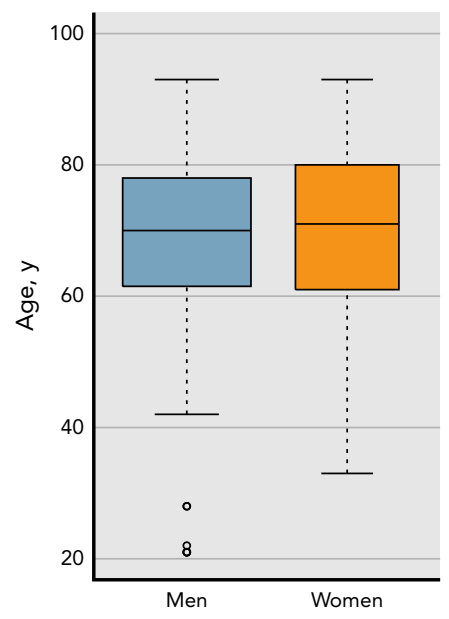

D

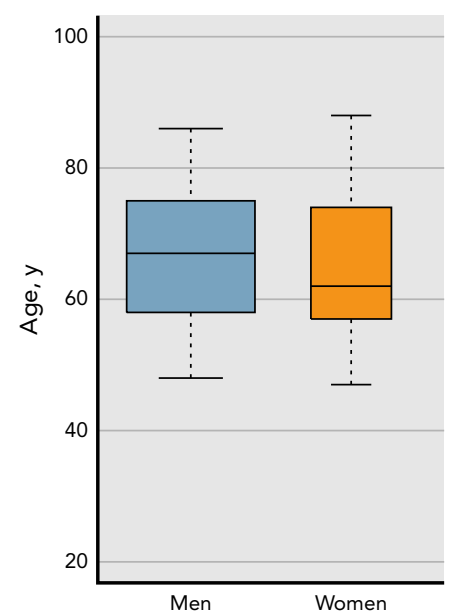

B

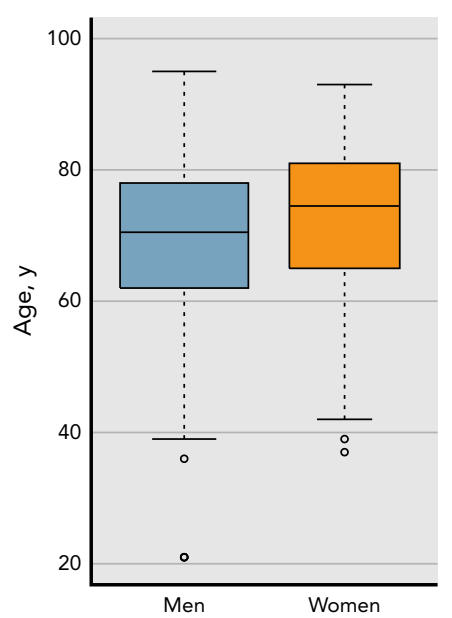

E

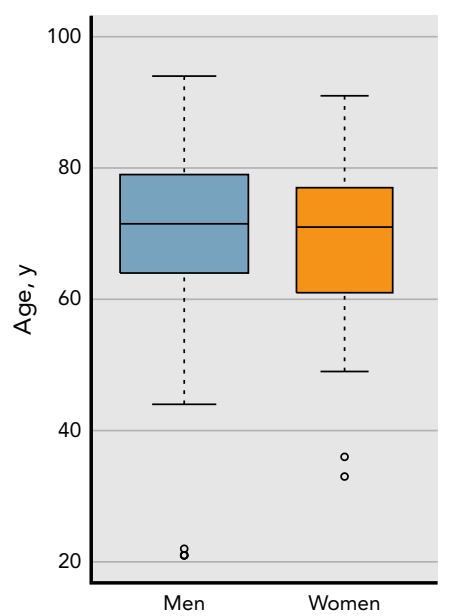

C

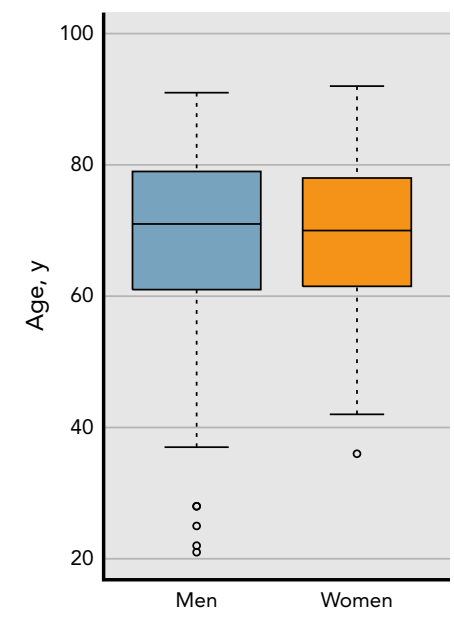

$\mathbf{F}$

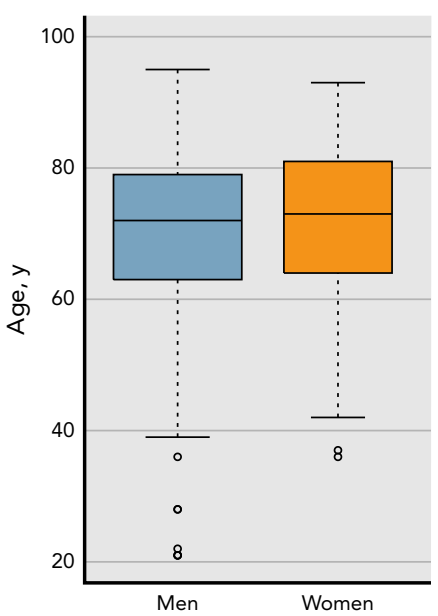

Figure 2. Age at diagnosis according to metastatic site and sex in patients with metastatic upper tract urothelial carcinoma and (A) lung metastases, (B) abdominal metastases, (C) lymph node metastases, (D) brain metastases, (E) bone metastases, and (F) $>1$ metastatic site.

symptoms or in patients with laboratory indicators of bone metastases. Moreover, the NCCN recommendation regarding bone imaging was based on a lower level of evidence (category 2A) with uniform consensus among the NCCN Bladder Cancer Panel that the intervention is appropriate. ${ }^{11}$ Conversely, the European Association of Urology guidelines ${ }^{8}$ do not mention bone imaging at all but rely on CT scan as the preferred diagnostic technique for lung and abdominal staging of metastases.

Second, we identified a low rate of brain metastases in both sexes ( $4.2 \%$ of men vs $2.4 \%$ of women), suggesting that routine brain imaging may not be required, unless it is based on an elevated level of clinical suspicion. This approach is consistent with contemporary guidelines. ${ }^{8,11}$

Third, in sex-stratified analyses, we identified differences in rates of bone metastases, which were $22.3 \%$ in men versus $18.0 \%$ in women. Conversely, the opposite difference in rates was recorded for liver metastases ( $24.4 \%$ of women vs $20.5 \%$ of men). Moreover, ovarian metastases may exist in women $(2.2 \%)$. No additional clinically meaningful differences in sex distribution were identified. Notably, none of the sex-related differences achieved statistical significance.

Despite the presence of sex-related differences in the distribution of bone and liver metastases, these differences have little, if any, impact on everyday clinical practice; however, they may be important in planning and design of clinical trials. ${ }^{12-14}$ For example, studies addressing bone-targeted agents may require consideration of sexrelated differences for the purpose of study design. ${ }^{15}$

Fourth, in age- and sex-stratified analyses of exclusive metastatic site distribution, we identified only one 


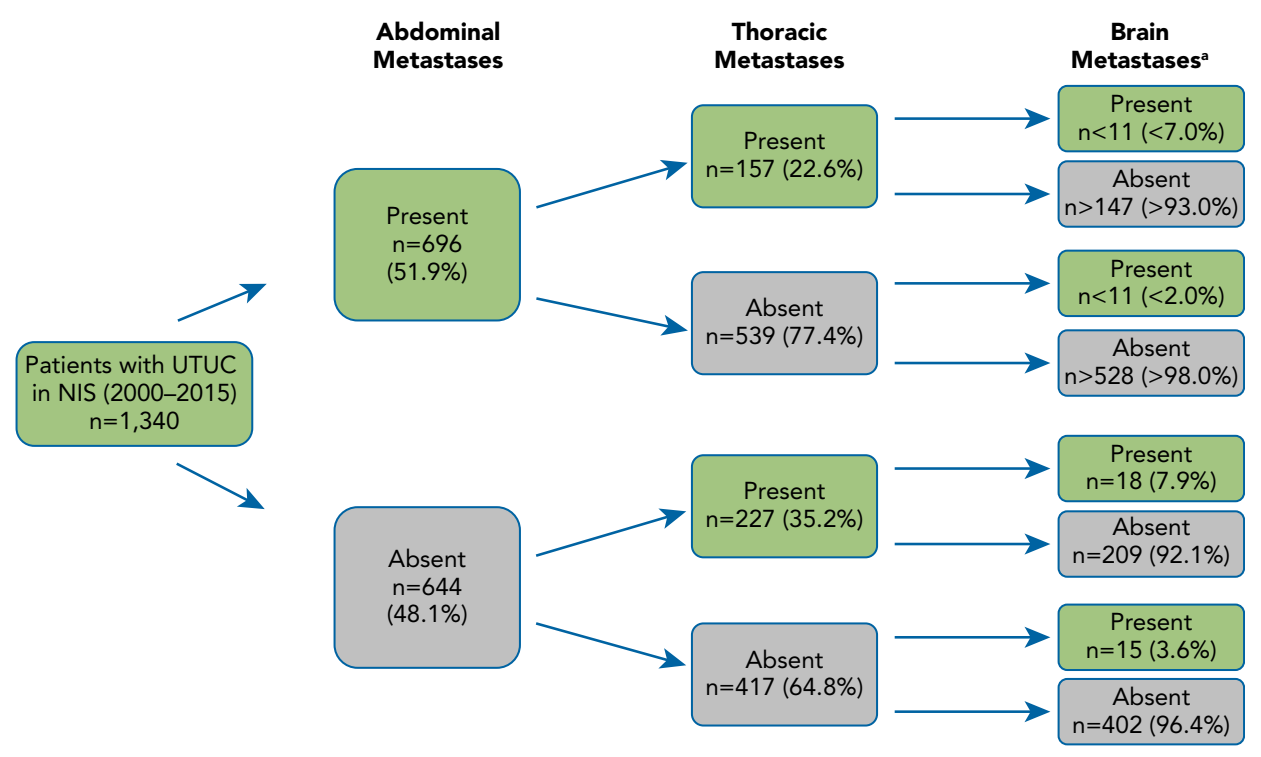

Figure 3. Rates of conditional brain metastases according to absence or presence of abdominal and thoracic metastases. Abbreviations: NIS, Nationwide Inpatient Sample; UTUC, upper tract urothelial carcinoma.

aPatient counts $\leq 10$ cannot be displayed to adhere to privacy protection requirements of Healthcare Cost and Utilization Project data.

clinically meaningful and statistically significant observation in 4 sets of analyses that focused on lung, bone, liver, and brain. Specifically, the rate of exclusive liver metastases increased with increasing age in female patients. This observation may be of assistance to clinicians who suspect liver metastases. Our findings indicate that the index of suspicion doubles with increasing age (from $21 \%$ to $41 \% ; P=.04$ ). The presence of this trend is helpful; however, it is not sufficiently specific to rule in or out liver metastases.

Fifth, based on a stratified conditional approach, brain metastases were invariably infrequent. Therefore, the presence of multiple metastases within the abdomen and/or thorax is not a reliable indicator of brain metastases. Thus, brain imaging without clinical suspicion of brain metastases may not be warranted, even in patients with combined abdominal and thoracic metastases. Moreover, the absence of metastases in the abdomen and thorax does not in any way rule out the possibility of brain metastases, and its presence should still be considered based on clinical suspicion.

Notably, our study represents the first populationbased effort aimed at tabulating and describing the metastatic sites of UTUC according to age and sex. Only 2 other studies addressed the same topic, and they were based on 52 and 337 patients, respectively. ${ }^{2,3}$ Like our study, the studies by Shinagare et $\mathrm{al}^{2}$ and Dong et $\mathrm{al}^{3}$ found the lung to be the most prevalent site of metastasis, whereas the liver was more common than bone in the analyses by Shinagare et al, and Dong et al found the same rates for bone and liver metastases. Like our study, their studies exclusively focused on patients with metastatic disease and did not provide a denominator, nor could they quantify the rates of de novo metastatic UTUC. Therefore, incidence data regarding de novo metastatic UTUC are still needed. Unfortunately, the current database could not provide such data. Only one older population-based study (1973-2005) addressed incidence rates of UTUC, reporting that $8 \%$ of patients with UTUC presented with a distant metastatic stage, ${ }^{16}$ but the study was without closer stratification regarding sites of metastases.

Considering all of the findings together, the present study provides the first contemporary description of detailed distribution of UTUC metastases according to age and sex. Our findings indicate that routine bone surveys should be universally performed in patients with UTUC because bone metastases represent the second and fourth commonest sites in men and women, respectively. Conversely, brain imaging may continue to be performed in select patients based on clinical suspicion for brain metastases. In addition, we identified sex-specific differences in bone and liver metastases and a sex- and age-specific increase in solitary liver metastases in women. However, the magnitude of these sexand age-related differences was too small for application in everyday clinical practice. Nonetheless, these differences may be of importance in the planning and design of clinical trials.

Despite its novelty, our study is not devoid of limitations. First, the NIS database includes only hospitalized 
patients, and therefore, patients who never required hospitalization have been omitted from our analyses. Second, the NIS is an observational, retrospective database relying on ICD-9 codes to assess secondary diagnostic codes, which may be subject to potential coding biases. Moreover, the NIS database does not provide laboratory results or other comorbidity information. Specifically, histologic subgroups, such as urothelial versus variant histology, could not be assessed because of design limitations of the database. Nonetheless, the NIS estimates are considered to be precise and accurate. ${ }^{6}$ Finally, our analyses focused exclusively on a cohort with metastases and thus cannot provide incidence rates of metastatic sites in the overall UTUC population.

\section{Conclusions}

Lung, bone, and liver metastases are the most common metastatic sites in both sexes. However, the distribution of metastases varies according to sex and age. These observations apply to everyday clinical practice and may be used, for example, to advocate for universal bone imaging in patients with UTUC. Moreover, our findings may also be used in design considerations of randomized trials.

Submitted March 6, 2020; accepted for publication August 9, 2020. Published online February 11, 2021.

Author contributions: Study concept and design: Deuker, Stolzenbach, Collà Ruvolo, Nocera, Martin, Tian, Karakiewicz. Data acquisition: Tian, Karakiewicz. Quality control of data and algorithms: Deuker, Tian. Data analysis and interpretation: Deuker, Stolzenbach, Collà Ruvolo, Nocera, Martin, Tian. Statistical analysis: Deuker, Tian. Manuscript preparation: Deuker, Stolzenbach, Collà Ruvolo, Nocera, Martin. Manuscript editing: Roos, Becker, Kluth, Tilki, Shariat, Saad, Chun, Karakiewicz. Manuscript review: Chun, Karakiewicz. Disclosures: The authors have disclosed that they have not received any financial consideration from any person or organization to support the preparation, analysis, results, or discussion of this article.

Correspondence: Marina Deuker, MD, Department of Urology, University Hospital Frankfurt, Theodor-Stern Kai 7, 60590 Frankfurt am Main, Germany. Email: marina.deuker@kgu.de

\section{References}

1. Munoz JJ, Ellison LM. Upper tract urothelial neoplasms: incidence and survival during the last 2 decades. J Urol 2000;164:1523-1525.

2. Shinagare $A B$, Fennessy FM, Ramaiya $\mathrm{NH}$, et al. Urothelial cancers of the upper urinary tract: metastatic pattern and its correlation with tumor histopathology and location. J Comput Assist Tomogr 2011;35:217-222.

3. Dong $F$, Fu H, Shi $X$, et al. How do organ-specific metastases affect prognosis and surgical treatment for patients with metastatic upper tract urothelial carcinoma: first evidence from population based data. Clin Exp Metastasis 2017;34:467-477.

4. Stolzenbach LF, Rosiello G, Deuker M, et al. The impact of race and age on distribution of metastases in patients with prostate cancer. J Urol 2020; 204:962-968.

5. Bianchi M, Roghmann F, Becker A, et al. Age-stratified distribution of metastatic sites in bladder cancer: a population-based analysis. Can Urol Assoc J 2014;8:E148-158.

6. HCUP Databases. Healthcare Cost and Utilization Project (HCUP). Agency for Healthcare Research and Quality, Rockville, MD. Accessed December 20, 2020. Available at: www.hcup-us.ahrq.gov/databases.jsp

7. Bianchi M, Sun M, Jeldres $C$, et al. Distribution of metastatic sites in renal cell carcinoma: a population-based analysis. Ann Oncol 2012;23:973-980.

8. Rouprêt M, Babjuk M, Burger M, et al. European Association of Urology guidelines on upper urinary tract urothelial carcinoma: 2020 update. Eur Urol 2021;79:62-79.

9. Pianko MJ, Terpos E, Roodman GD, et al. Whole-body low-dose computed tomography and advanced imaging techniques for multiple myeloma bone disease. Clin Cancer Res 2014;20:5888-5897.

10. Heindel W, Gübitz R, Vieth $V$, et al. The diagnostic imaging of bone metastases. Dtsch Arztebl Int 2014;111:741-747.

11. Flaig TW, Spiess PE, Agarwal N, et al. NCCN Clinical Practice Guideline in Oncology: Bladder Cancer. Version 6.2020. Accessed December 10, 2020. To view the most recent version, visit NCCN.org

12. Hussain SA, Birtle A, Crabb S, et al. From clinical trials to real-life clinical practice: the role of immunotherapy with PD-1/PD-L1 inhibitors in advanced urothelial carcinoma. Eur Urol Oncol 2018;1:486-500.

13. Petrylak DP, de Wit R, Chi KN, et al. Ramucirumab plus docetaxel versus placebo plus docetaxel in patients with locally advanced or metastatic urothelial carcinoma after platinum-based therapy (RANGE): a randomised, double-blind, phase 3 trial. Lancet 2017;390:2266-2277.

14. Anari F, O'Neill J, Choi W, et al. Neoadjuvant dose-dense gemcitabine and cisplatin in muscle-invasive bladder cancer: results of a phase 2 trial. Eur Urol Oncol 2018;1:54-60.

15. Zaghloul MS, Boutrus $\mathrm{R}, \mathrm{El}-\mathrm{H}$ ossieny $\mathrm{H}$, et al. A prospective, randomized placebo-controlled trial of zoledronic acid in bony metastatic bladder cancer. Int J Clin Oncol 2010;15:382-389.

16. Raman JD, Messer J, Sielatycki JA, et al. Incidence and survival of patients with carcinoma of the ureter and renal pelvis in the USA, 1973-2005. BJU Int 2011;107:1059-1064. 
Supplemental online content for:

\section{Sex- and Age-Related Differences in the Distribution of Metastases in Patients With Upper Urinary Tract Urothelial Carcinoma}

Marina Deuker, MD; Giuseppe Rosiello, MD; Lara Franziska Stolzenbach, MD; Thomas Martin, MD; Claudia Collà Ruvolo, MD; Luigi Nocera, MD; Zhe Tian; Frederik C. Roos, MD; Andreas Becker, MD; Luis A. Kluth, MD; Derya Tilki, MD; Shahrokh F. Shariat, MD; Fred Saad, MD; Felix K.H. Chun, MD; and Pierre I. Karakiewicz, MD

J Natl Compr Canc Netw 2021;19(5):534-540

eFigure 1: CONSORT Diagram Depicting Patients Excluded From Analysis 


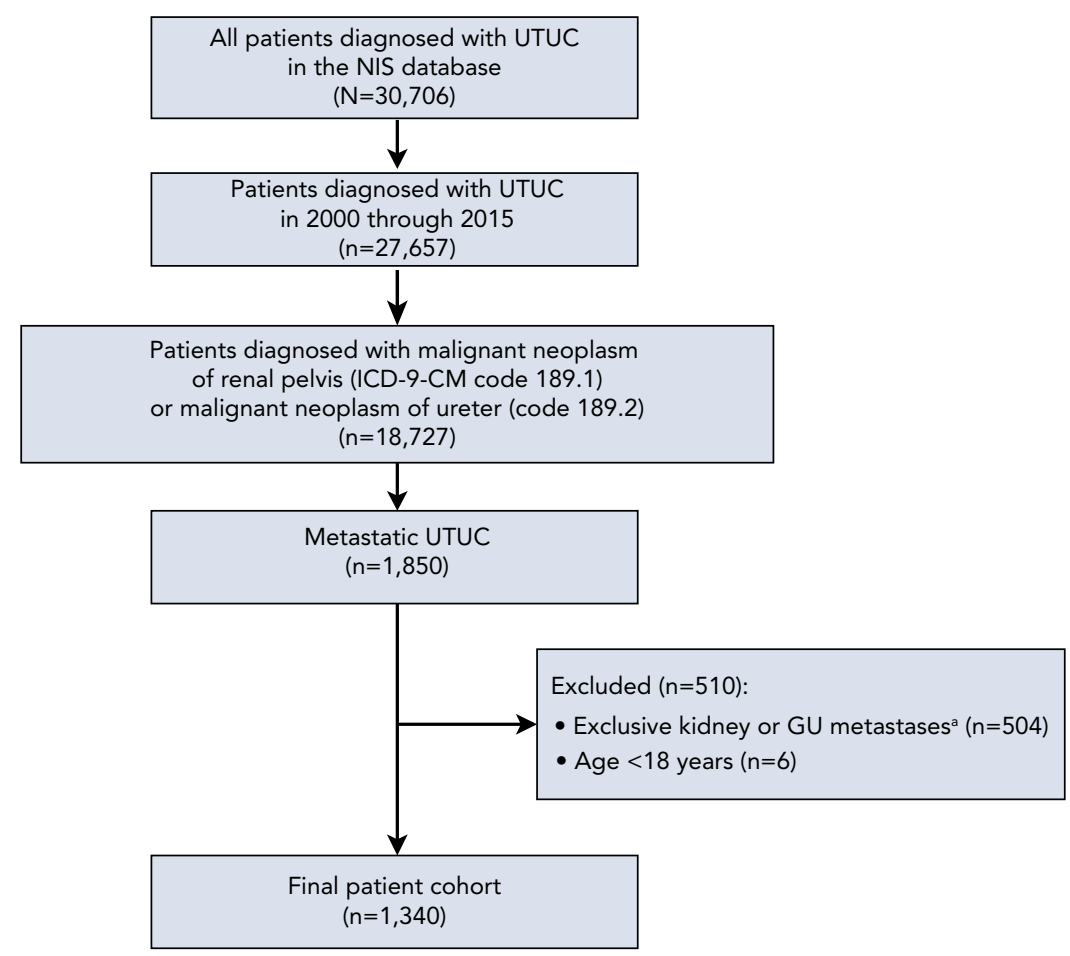

eFigure 1. CONSORT diagram of patient selection.

Abbreviations: GU, genitourinary; NIS, Nationwide Inpatient Sample; UTUC, upper tract urothelial carcinoma.

aBased on the notion that exclusive kidney or exclusive GU metastases encompass concomitant primary tumor sites rather than real metastases, because these metastases commonly occur in urothelial cancer at several locations in the urinary tract without being considered metastatic. 